
\section{(PRECA! TECH}

Determination of microplastic content in freshwater lakes in

\section{Introduction}

Studies have shown that plastics degrade into microplastics with a diameter of $5 \mathrm{~mm}$ or less. Many studies have been completed on the presence of microplastics in marine environments, with fewer studies on freshwater environments. This study attempts to determine the content of microplastics in two freshwater lakes in Tennessee that have varying degrees of human activity near the lake Kentucky Lake on the Tennessee River and Maple Creek Lake isolated in the Natchez Trace State Park. Samples were collected from various locations within the two lakes via grab sampling. The size of the microplastics were determined with a particle size analyzer, and then the chemical composition of the particles was determined by FT-IR

\section{Sampling Locations}

Samples were taken from three locations along Kentucky Lake on the Tennessee River and two locations on Maple Creek Lake in Carroll County, Tennessee. A sample was taken at each of the three Kentucky Lake locations on Saturday, August 31, 2019 prior to large scale recreational aquatic activities as part of Labor Day weekend. A second sample was taken at the same locations on the evening of Monday, September 2, 2019. Both samples were taken on Maple Creek Lake on Monday, September 2, 2019

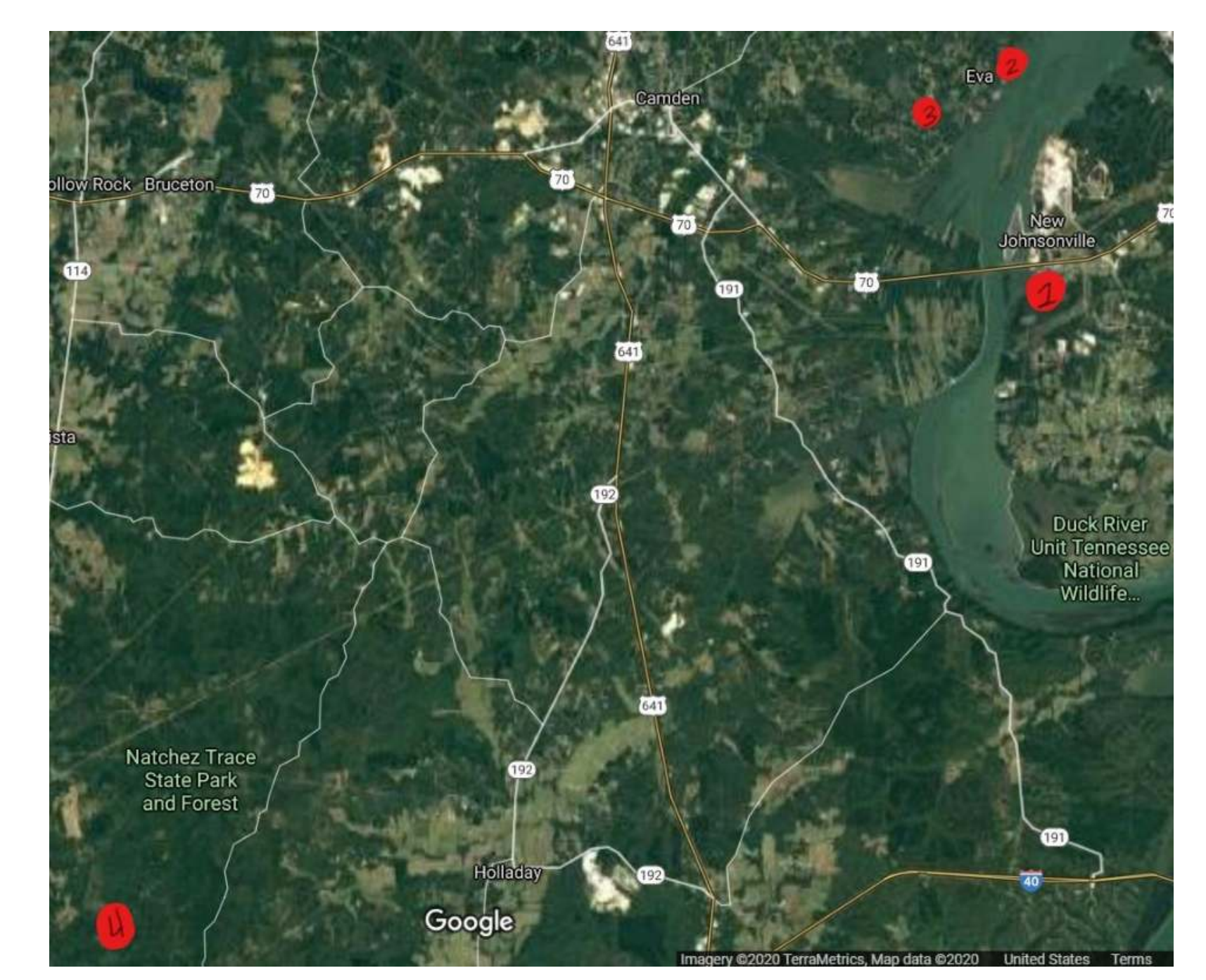

Figure 1. Satellite Map image with marked locations of where samples were taken. Image credit to Google,
TerraMeterics, Map Data
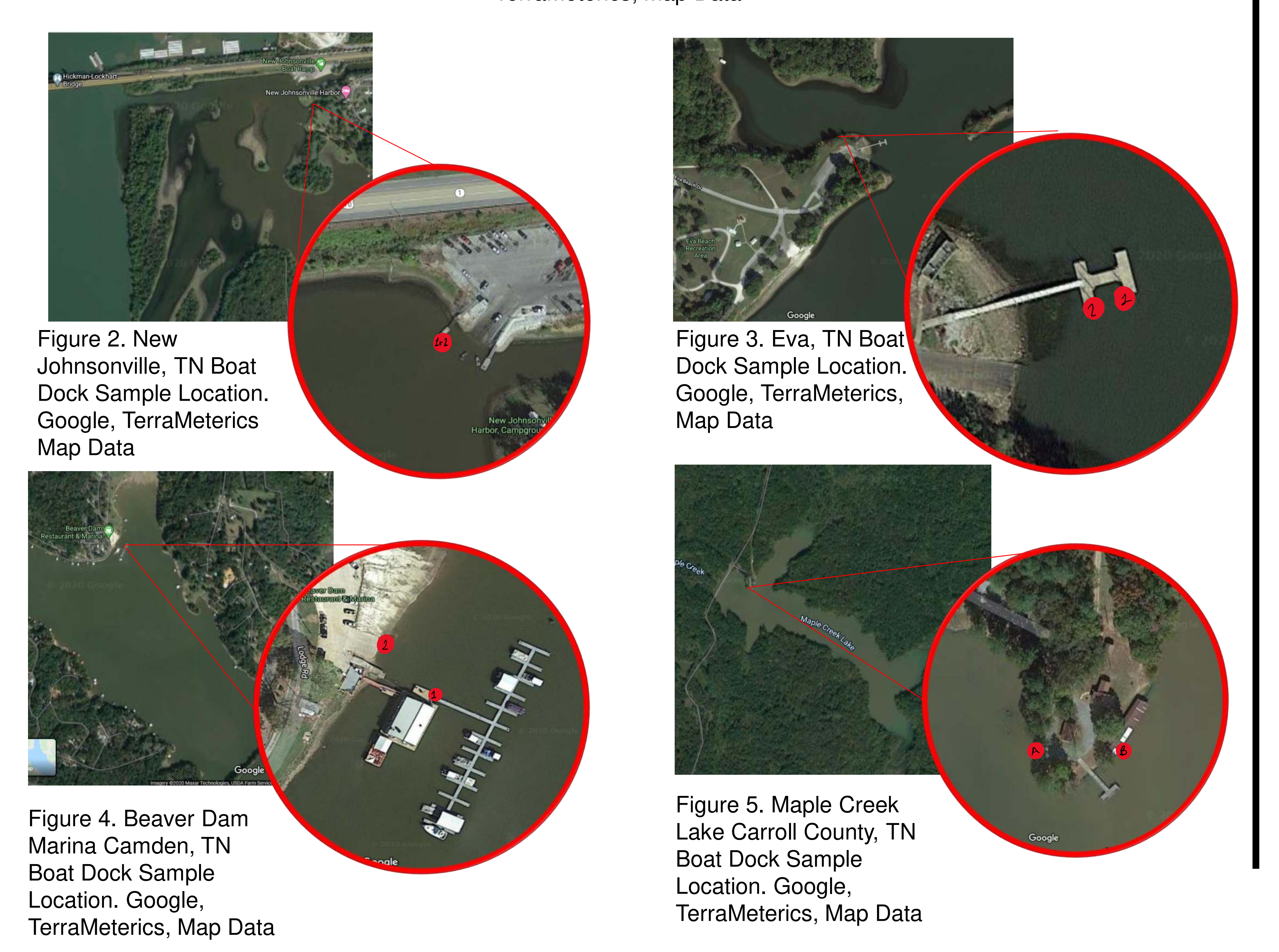

western Tennessee

Rachel Baker, Chioma Onwuchekwa (Ekechi), and Tammy H. Boles, PhD

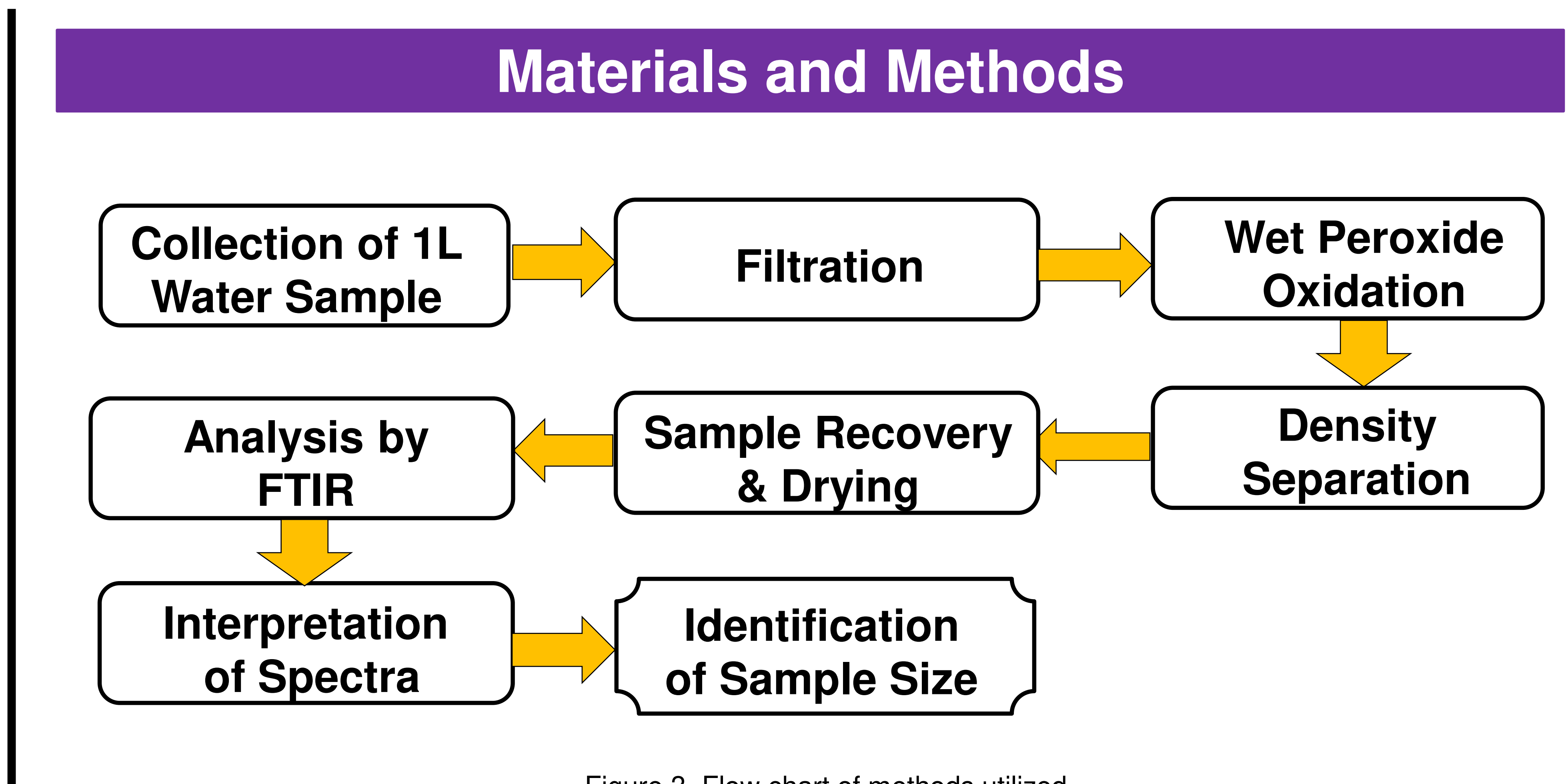

Samples were collected in $1 \mathrm{~L}$ glass bottles from each sample location. Each sample was filtered through three sieves with mesh sizes of $5 \mathrm{~mm}, 1 \mathrm{~mm}$, and $0.3 \mathrm{~mm}$. After filtering, particles were collected from the mesh of the $1 \mathrm{~mm}$ and $0.3 \mathrm{~mm}$ sieves and washed into a beaker with ethanol. After evaporation of the ethanol, the retained particles were treated with wet peroxide oxidation to remove any remaining biological material from the sample. $\mathrm{NaCl}$ was added to the sample beaker after the oxidation was complete to create a density difference which allowed any plastic particles to float at the top of the sample. Any microplastics found in the sample were removed from the density separation apparatus, and allowed to dry. The recovered microplastics were identified using PerkinElmer FT-IR in ATR mode.

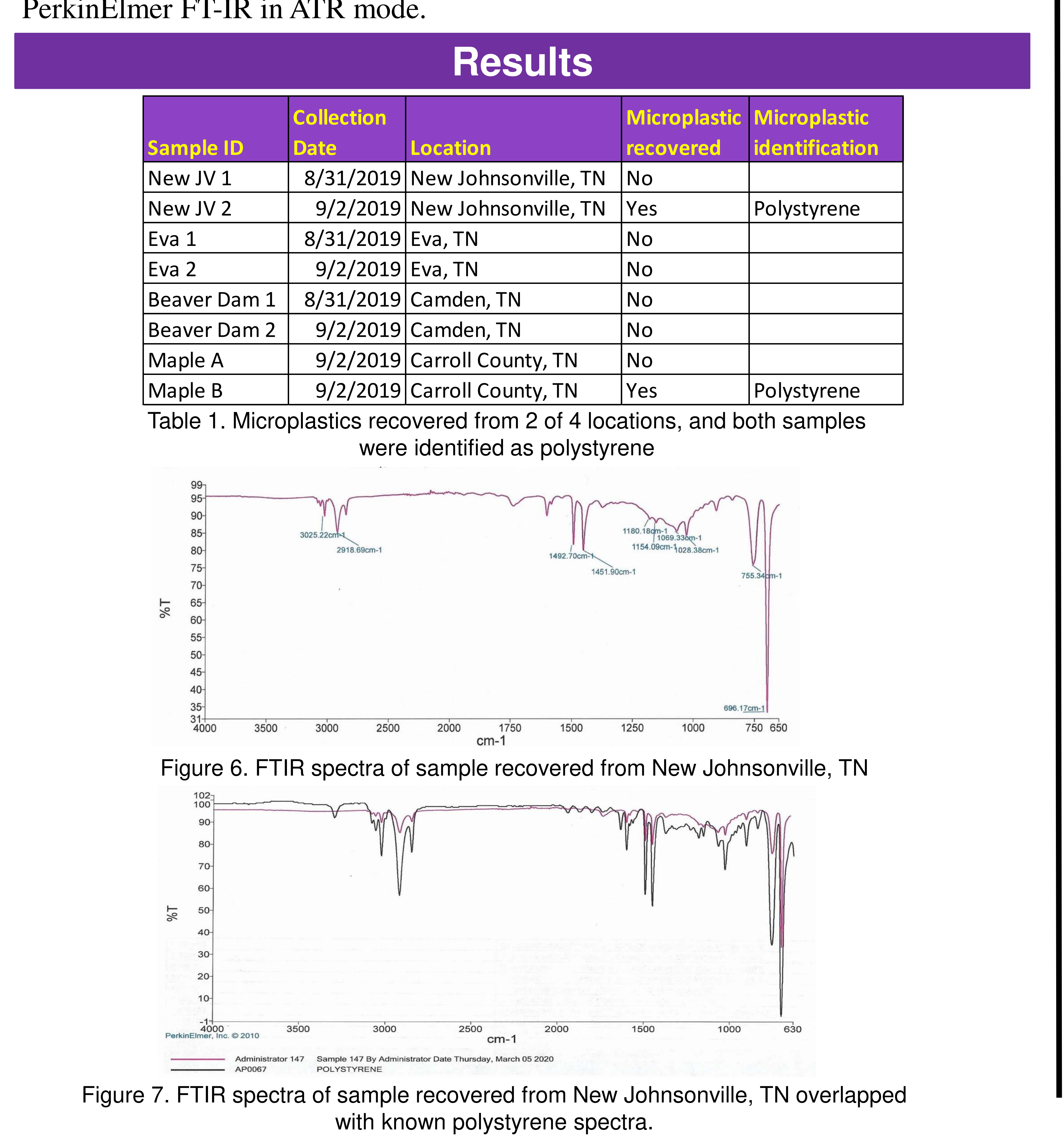

School of Environmental Studies College of Interdisciplinary Studies

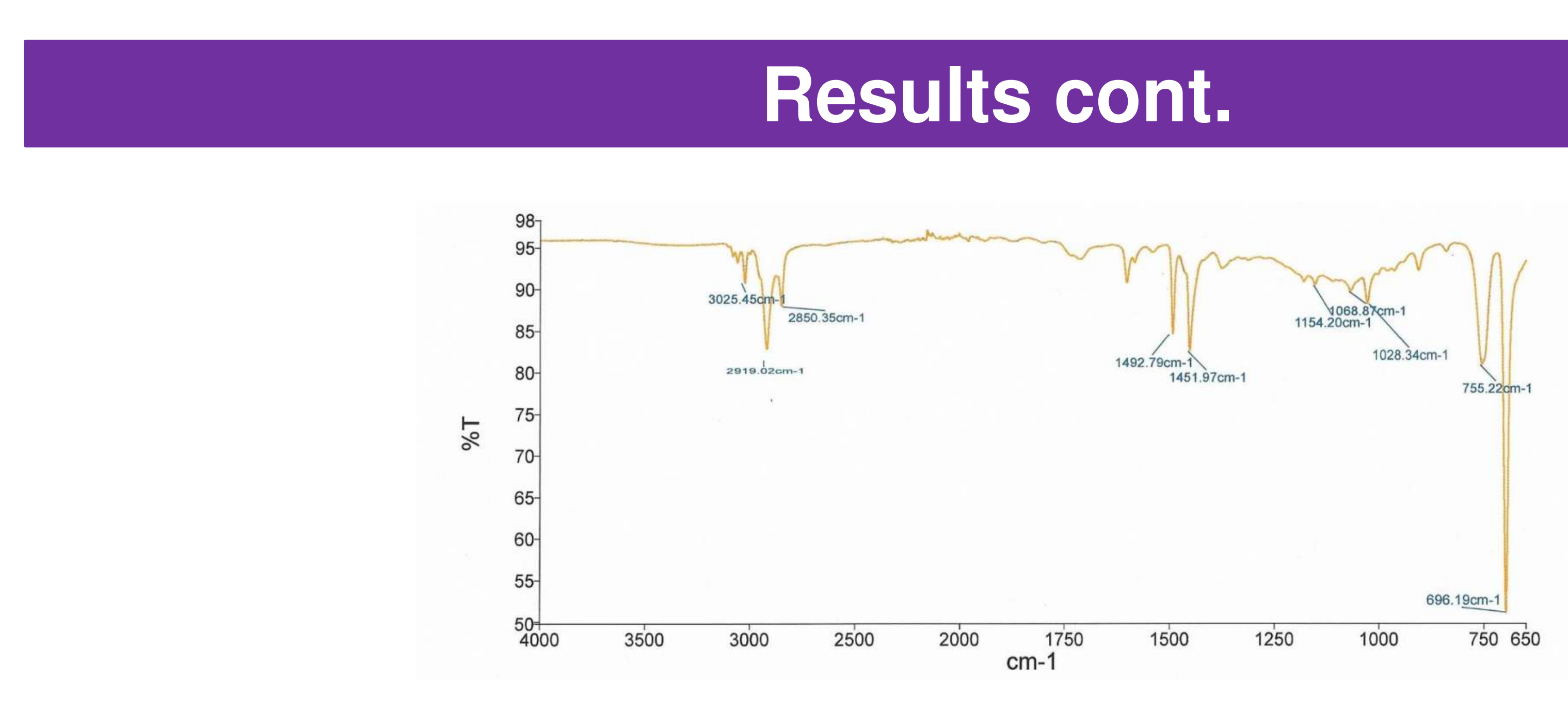

Figure 8. FTIR spectra of sample B recovered from Maple Creek Lake

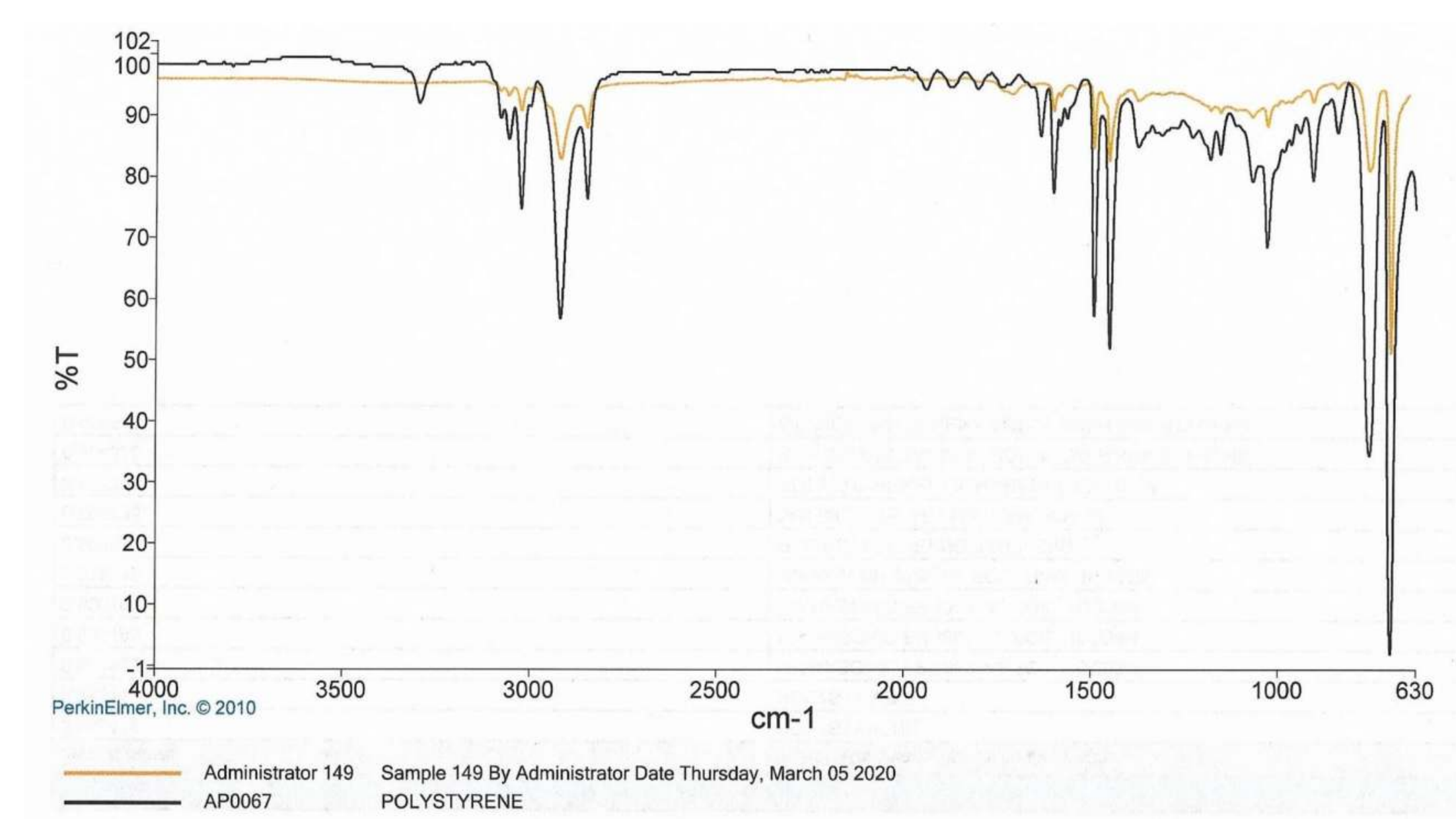

Figure 9. FTIR spectra of sample recovered from Maple Creek Lake overlapped with known polystyrene spectra.

Results show that two of the eight samples taken from western TN lakes contain microplastics in the size range greater than $0.3 \mathrm{~mm}$ and less than or equal to $5 \mathrm{~mm}$. The analysis of the FT-IR spectra indicate the recovered samples were polystyrene. These results are indicative that both Kentucky Lake and Maple Creek Lake contain microplastic in the aquatic environment.

\section{Future Research}

There is need for more research and analysis to be completed. The samples analyzed were collected by grab sampling, future studies should be completed on samples collected by trawling a larger are of the aquatic environments of interest.

\section{Selected References}

Arthur, C., Bamford, H. \& Baker, J. (2009) 'Proceedings of the International Research Workshop on the Occurrence, Effects and Fate of Microplastic Marine Debris. Sept 9-

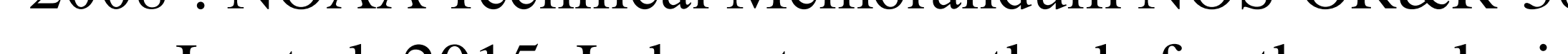

Masura, J., et al. 2015. Laboratory methods for the analysis of microplastics in the marine sediments. NOAA Technical Memorandum NOS-OR\&R-48.

Song. Y. K · Hong. S. H. Jang. M. Kang J.-H. Kwon O. Y. Han, G. M - Shim, W. J. Large Accumulation of Micro-Sized Synthetic Polymer Particles in the Sea Surface Microlayer. Environ. Sci. Technol. 2014, 48 (16), 9014-9021.

https://doi.org/10.1021/es501757s.

\section{Acknowledgements}

We wish to thank Tennessee Tech University, the School of Environmental Studies, and the Department of Chemistry for funding and support of this research. I would also like to thank the TTU URECA! Program 